\title{
Mucolipidosis types II and III and non-syndromic stuttering are associated with different variants in the same genes
}

\author{
M Hashim Raza ${ }^{1}$, Carlos EF Domingues ${ }^{1}$, Ronald Webster ${ }^{2}$, Eduardo Sainz ${ }^{1}$, Emily Paris ${ }^{1,6}$, Rachel Rahn ${ }^{1}$, \\ Joanne Gutierrez ${ }^{1}$, Ho Ming Chow ${ }^{1}$, Jennifer Mundorff ${ }^{2}$, Chang-soo Kang ${ }^{1,7}$, Naveeda Riaz ${ }^{3}$, \\ Muhammad AR Basra ${ }^{4,8}$, Shaheen $\mathrm{Khan}^{4}$, Sheikh Riazuddin ${ }^{4}$, Danilo Moretti-Ferreira ${ }^{5}$, Allen Braun ${ }^{1}$ and \\ Dennis Drayna $^{*, 1}$
}

\begin{abstract}
Homozygous mutations in GNPTAB and GNPTG are classically associated with mucolipidosis II (ML II) alpha/beta and mucolipidosis III (ML III) alpha/beta/gamma, which are rare lysosomal storage disorders characterized by multiple pathologies. Recently, variants in GNPTAB, GNPTG, and the functionally related NAGPA gene have been associated with non-syndromic persistent stuttering. In a worldwide sample of 1013 unrelated individuals with non-syndromic persistent stuttering we found 164 individuals who carried a rare non-synonymous coding variant in one of these three genes. We compared the frequency of these variants with those in population-matched controls and genomic databases, and their location with those reported in mucolipidosis. Stuttering subjects displayed an excess of non-synonymous coding variants compared to controls and individuals in the 1000 Genomes and Exome Sequencing Project databases. We identified a total of 81 different variants in our stuttering cases. Virtually all of these were missense substitutions, only one of which has been previously reported in mucolipidosis, a disease frequently associated with complete loss-of-function mutations. We hypothesize that rare non-synonymous coding variants in GNPTAB, GNPTG, and NAGPA may account for as much as $16 \%$ of persistent stuttering cases, and that variants in GNPTAB and GNPTG are at different sites and may in general, cause less severe effects on protein function than those in ML II alpha/beta and ML III alpha/beta/gamma.
\end{abstract}

European Journal of Human Genetics (2016) 24, 529-534; doi:10.1038/ejhg.2015.154; published online 1 July 2015

\section{INTRODUCTION}

Stuttering is a common disorder characterized by involuntary disruptions in the flow of speech that has been shown to have strong genetic contributions. ${ }^{1-5}$ The disorder has been associated with variants in GNPTAB (encoding $N$-acetylglucosamine-1-phosphotransferase alpha/ beta subunits precursor), GNPTG (encoding $N$-acetylglucosamine-1phosphotransferase gamma subunit), and NAGPA (encoding $\mathrm{N}$-acetylglucosamine-1-phosphodiester alpha- $\mathrm{N}$-acetylglucosaminidase). ${ }^{6}$ In the initial report, $6.3 \%$ of unrelated subjects with persistent stuttering carried a variant in one of these three genes. ${ }^{6}$ Using a larger sample we have re-examined the frequency of rare nonsynonymous coding variants in these genes in this disorder. Variants in GNPTAB and GNPTG are classically associated with mucolipidosis II (ML II) alpha/beta (OMIM \#252500), mucolipidosis III (ML III) alpha/beta (OMIM \#252600), and mucolipidosis III (ML III) gamma (OMIM \#252605), which are rare, severe lysosomal storage disorders caused by homozygous mutations in these genes. We also compared the rare coding variants observed in GNPTAB and GNPTG in stuttering subjects with those reported in mucolipidosis patients.

\section{MATERIALS AND METHODS}

Unrelated individuals with persistent developmental stuttering and no other neurological conditions were enrolled in the United States and England (HCRI $+\mathrm{NIH}$ and NAF series, 634 total subjects), Pakistan (PKST series, $N=124$ ), Cameroon (STCR series, $N=96$ ), and Brazil (BRCS series, $N=159$ ), and population/gender-matched controls from the United States (NDPT series, $N=276$ ), Pakistan (RPP series, $N=96$ ), Cameroon (RC series, $N=94$ ), and Brazil (BRCO series, $N=211$ ) with written informed consent under NIH protocol \#97-DC-0057, as previously described. ${ }^{6}$ The DNAs from North American subjects determined to be normal following extensive neurological examination were obtained from spouse controls in a large population-based study of Parkinson's Disease (National Institute of Neurological Disorders and Stroke (NINDS) panels NDPT006, -020, -023, -079, -082 and -093) from the Coriell Cell Repository. Extensive clinical data on these subjects and access to these DNAs is available at http://ccr.coriell.org/Sections/Collections/NINDS/ DNAPanels.aspx?PgId $=195 \&$ coll $=$ ND. All affected subjects reported a family history of stuttering. Sequences from large population samples were obtained from the 1000 Genomes Project database (http://www.1000genomes.org) and the NHLBI Exome Sequencing Project (ESP, comprising 6400 exomes) (http://evs.gs.washington.edu/EVS/). Variants associated with ML II alpha/beta

\footnotetext{
${ }^{1}$ Laboratory of Communication Disorders, National Institute on Deafness and Other Communication Disorders, National Institutes of Health, Porter Neuroscience Research Center, Bethesda, MD, USA; ${ }^{2}$ Hollins Communications Research Institute, Roanoke, VA, USA; ${ }^{3}$ Department of Bioinformatics and Biotechnology, International Islamic University, Islamabad, Pakistan; ${ }^{4}$ Department of Molecular Biology, Allama Iqbal Medical College, University of Health Sciences, Lahore, Pakistan; ${ }^{5}$ Department of Genetics, Sao Paulo State University, Botucatu, Brazil

${ }^{*}$ Correspondence: Dr D Drayna, Laboratory of Communication Disorders, National Institute on Deafness and Other Communication Disorders, National Institutes of Health, Porter Neuroscience Research Center, 35A Convent Drive, Room 1F-127, Bethesda, MD 20892, USA. Tel: +1 3014024930 ; Fax: +1 301 480 8019; E-mail: drayna@nidcd.nih.gov ${ }^{6}$ Current address: Department of Civil and Environmental Engineering, Stanford University, Stanford, CA, USA.

${ }^{7}$ Current address: Department of Chemistry and Biology, Sungshin Women's University, Seoul, Korea.

${ }^{8}$ Current address: Institute of Chemistry, University of the Punjab, Lahore, Pakistan.

Received 6 February 2015; revised 21 May 2015; accepted 3 June 2015; published online 1 July 2015
} 


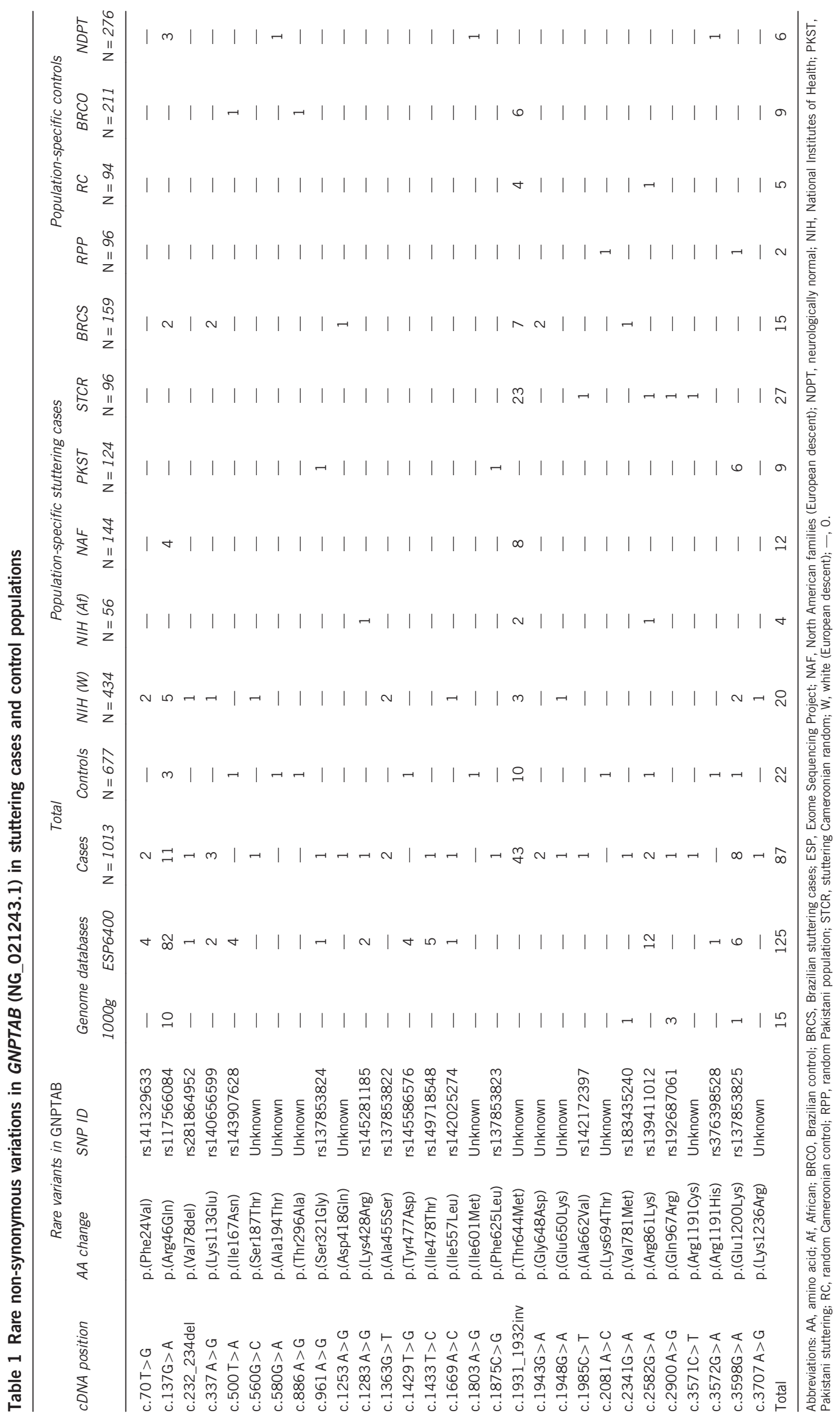




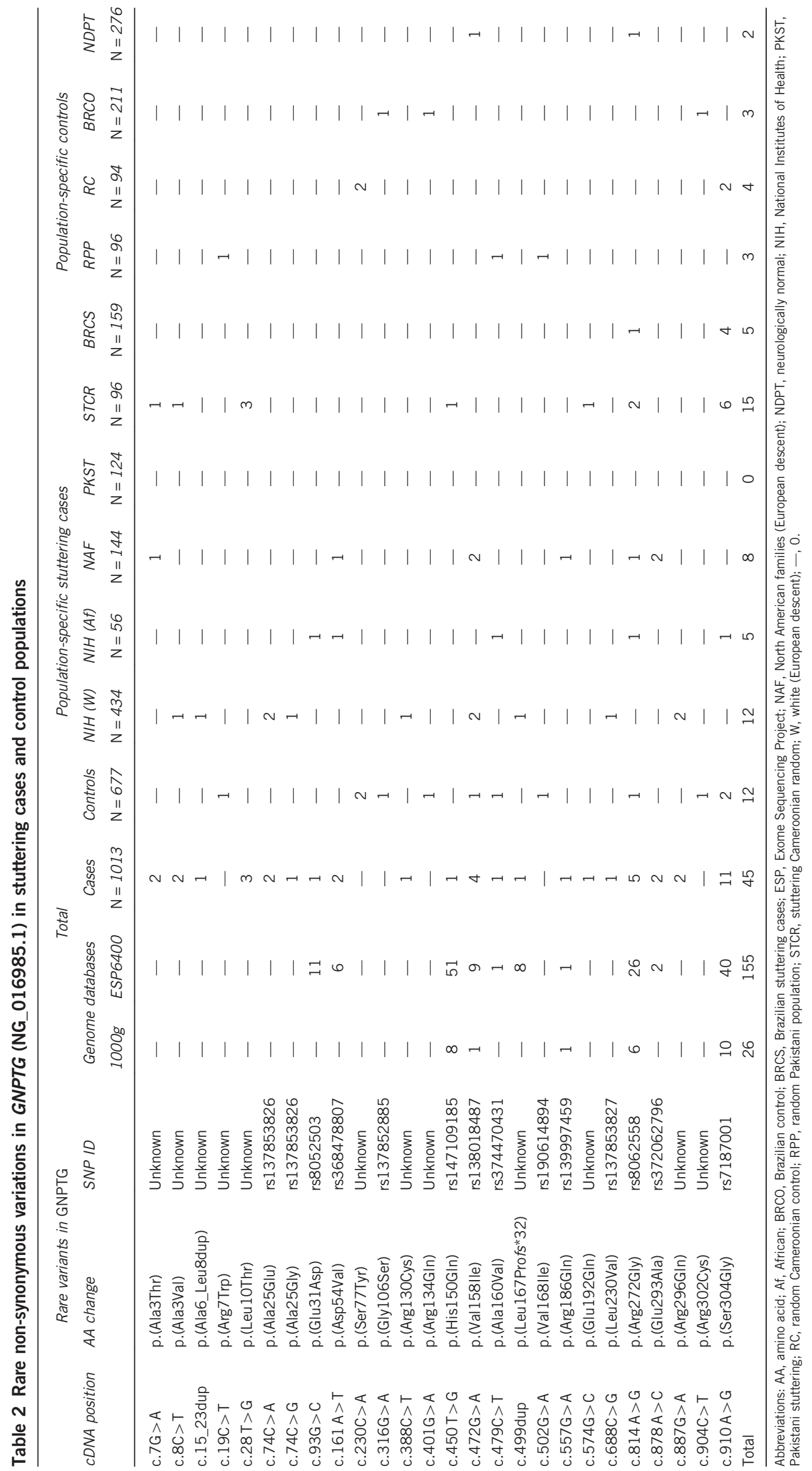




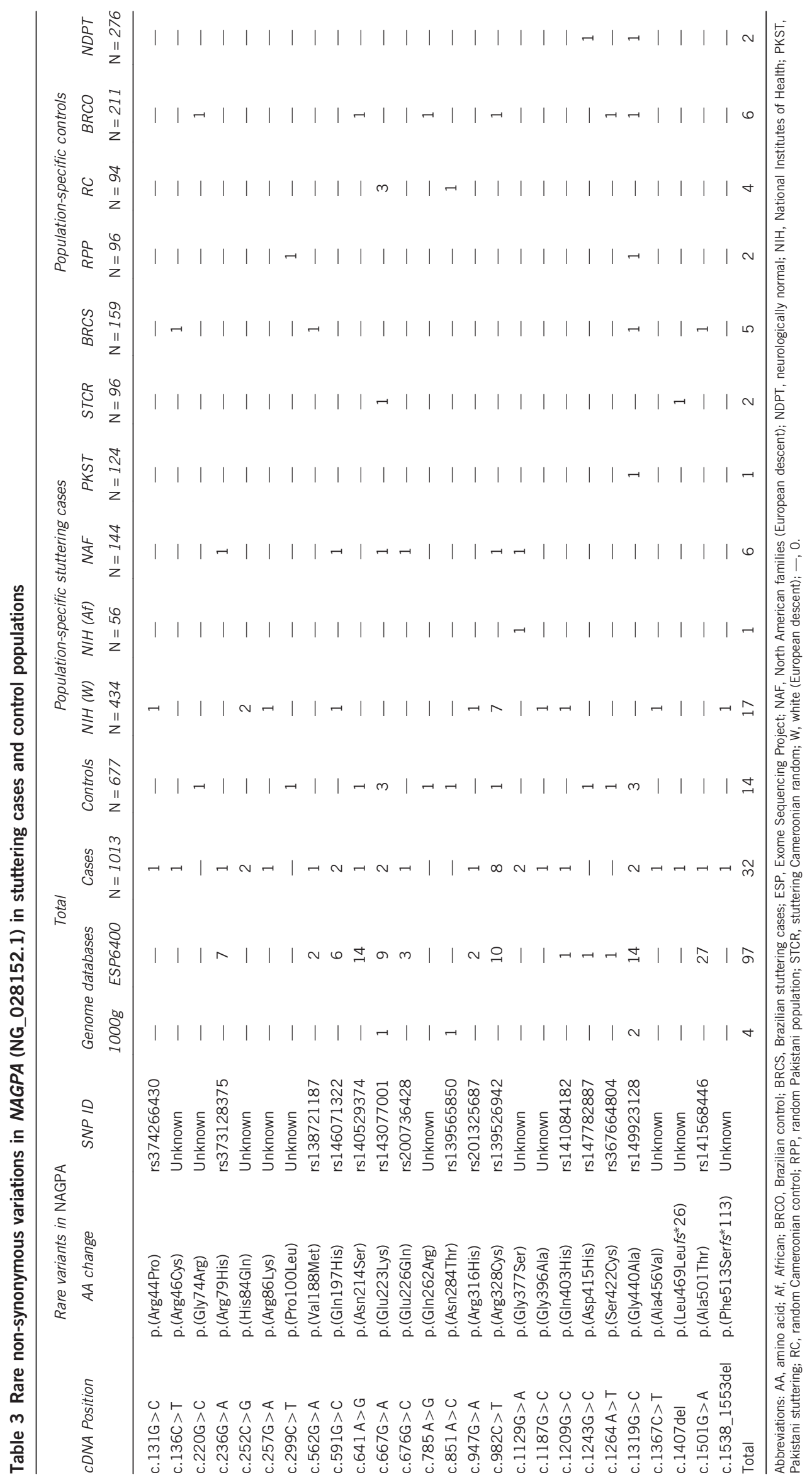


and ML III alpha/beta/gamma were obtained from the ClinVar database (http://www.ncbi.nlm.nih.gov/clinvar/?term=mucolipidosis), and from Cathey et al., Kudo et al., Liu et al., Paik et al., and Tiede et al. ${ }^{7-11}$

Stuttering diagnosis was performed using the Stuttering Severity Index-3 $(\text { SSI-3) })^{12}$ and as previously described. ${ }^{13}$ All subjects were unrelated by selfreport, and no genotypic evidence for relatedness among any subjects was observed.

Coding and adjacent intronic sequences of GNPTAB (NM_024312.4, NG_021243.1) GNPTG (NM_032520.4, NG_016985.1), and NAGPA (NM_016256.3, NG_028152.1) were amplified as previously described. ${ }^{6}$ Sequence variants were referenced from and have been deposited to the Locus Variation Data Base; http://databases.lovd.nl/shared/genes/GNPTAB for GNPTAB, http://databases.lovd.nl/shared/genes/GNPTG for GNPTG, and http://databases.lovd.nl/shared/genes/NAGPA for NAGPA.

\section{RESULTS}

Sequencing of the exons and flanking $\geq 50 \mathrm{bp}$ of intronic sequence of the GNPTAB, GNPTG, and NAGPA genes in unrelated individuals with familial persistent developmental stuttering identified many nonsynonymous coding variants. Variants that occurred at high frequency in cases and our population-matched controls were not considered further. In our previous studies, all of the stuttering-associated variants found in these three genes were rare coding sequence variants. ${ }^{6} \mathrm{We}$ therefore focused on non-synonymous coding sequence variants defined as rare by their presence at a frequency of 0.005 or less in the 1000 Genomes database. These variants are shown in Tables 1 (GNPTAB), 2 (GNPTG), and 3 (NAGPA). In our combined stuttering subject population (North American, Pakistani, Cameroonian, and Brazilian), such variants were observed in GNPTAB in 87 of 1013 cases, in GNPTG in 45 of 1013 cases, and in NAGPA in 32 of 1013 cases, for a total of 164 out of 1013 individuals (16\%). All carried a single copy of the variant. In total, these 164 subjects carried 81 different variants in these three genes. In contrast, we observed such variants in a total of 48 individuals out of 677 combined controls. This rate is significantly different from that observed in our combined cases sample $\left(\chi^{2}=30.63, P=3.13 \times 10^{-8}\right)$.

We also identified an excess of rare non-synonymous coding variants in these three genes in stuttering cases compared with the individuals in the 1000 Genomes database and Exome Sequencing Project database ( $\sim 400$ individuals), at a rate of $422 / 7400$ in these databases vs $164 / 1013$ in our cases $\left(\chi^{2}=151.21, P=9.42 \times 10^{-35}\right)$.

The excess of rare variants in cases compared with controls existed within all subpopulations in our sample, including those of European origin $\left(75 / 578\right.$ cases $v s$ 10/276 controls, $\left.\chi^{2}=18.23, P=1.9 \times 10^{-5}\right)$ and those of African ancestry including Brazilians, who have a large component of African ancestry, ${ }^{14}$ (79/311 cases vs 31/305 controls, $\left.\chi^{2}=24.4, P=7.93 \times 10^{-7}\right)$.

In addition to analyzing the sum of rare variants in cases and controls, we also examined the subset of variants that were observed in cases but not in controls, and compared these with variants observed in controls but not in cases. As shown in Tables 1-3, this subset comprised a total of 66 case subjects carrying variants that occurred in cases and not in controls, and 20 control subjects that carried a variant not seen in cases, a significant difference $\left(\chi^{2}=10.65, P=1.1 \times 10^{-3}\right)$.

We also surveyed rare variants in adjacent intronic sequences. We defined variants that could affect existing splice sites as those within $10 \mathrm{bp}$ of an exon, and identified a total of four such rare variants in our cases $(N=1013)$ in these three genes. All were present at similar frequencies in our neurologically normal controls, in our populationmatched control groups, and in the Exome Sequencing Project and 1000 Genomes databases.
With few exceptions, the coding variants in our stuttering cases were all missense amino-acid substitutions (Tables 1-3). A total of 81 mutations have been previously reported in ML II alpha/beta and ML III alpha/beta/gamma. ${ }^{7-11}$ None of these variants occurred in our stuttering case subjects with one exception, which was the $3 \mathrm{bp}$ inframe deletion in GNPTAB at amino-acid position 78 (Table 1). In particular, several mutations commonly observed in ML II alpha/beta and ML III alpha/beta/gamma, including the c.1399delG and c.3503_3504del frameshift mutations and the c.3335+6 T $>$ C splice site mutation in GNPTAB were not observed in our stuttering cases.

\section{DISCUSSION}

Our results confirm that rare non-synonymous coding variants in GNPTAB, GNPTG, and NAGPA are significantly more common in stuttering cases compared with controls and to the general populations, and provide an improved estimate of the contribution of mutations in these genes to persistent developmental stuttering. Our present estimate of $16 \%$ is substantially higher than the previous estimate of $6.3 \%$. However, we also identified rare variants that met our criteria in control subjects, albeit at a lower rate (7\%). If this $7 \%$ is considered the background rate of normal variation in these genes, then $16-7=9 \%$ of stuttering cases are attributable to variants in these three genes. However, perhaps as many as $1 \%$ of the individuals in the 1000 Genomes and ESP6400 databases are presumed to display stuttering to some degree, and as many as $5 \%$ had a history of childhood stuttering. ${ }^{15}$ In this case, rare coding variants in such individuals are not normal variation, and we hypothesize that the $16 \%$ would be an accurate estimate of the fraction of stuttering cases attributable to such variants.

Previous clinical studies of stuttering individuals who carry a mutation in GNPTAB, GNPTG, or NAGPA failed to reveal any symptoms of ML II alpha/beta and ML III alpha/beta/gamma, ${ }^{6}$ and it has been suggested that the mutations in GNPTAB and GNPTG that cause stuttering are fundamentally different than those that cause mucolipidosis. Our current results support this hypothesis. Only one of the 42 different variants in GNPTAB and GNPTG found in our stuttering subjects has been previously reported in mucolipidosis. In addition, more than 50 different mutations in GNPTAB and more than 30 different mutations in GNPTG have been identified in mucolipidosis patients ${ }^{7-11}$ (www.genecards.org). The majority of these mutations $(85 \%)$ are frameshifts, stop codons, deletions, or splice site mutations. ${ }^{7}$ Of the variants in GNPTAB, GNPTG, and NAGPA found in our stuttering subjects, $75 / 81$ (92.6\%) are missense substitutions, and the common mutations found in ML II and ML III were not observed. The one mucolipidosis mutation observed in our stuttering cases was not a missense mutation (the in-frame $3 \mathrm{bp}$ deletion encoding the valine at amino-acid position 78 in GNPTAB) and was observed in heterozygous state in one stuttering subject. This mutation has been previously reported homozygous in one case of ML III alpha/ beta/gamma (www.ncbi.nlm.nih.gov/clinvar/).

Our findings support the view that persistent stuttering is associated with mutations in GNPTAB or GNPTG that are generally not found in mucolipidosis and exert a less deleterious effect on protein function, and they provide an improved estimate of the contribution of mutations in these genes to stuttering.

\section{CONFLICT OF INTEREST}

The authors declare no conflict of interest. 


\section{ACKNOWLEDGEMENTS}

This research was supported by the NIDCD intramural grant \# Z1A-000046-14. We thank the Stuttering Foundation of America, the National Stuttering Association, the British Stammering Association, and the Speak Clear Association of Cameroon for assistance. We thank Linda Booth of HCRI for assistance in subject enrollment and sampling, and Drs Robert Morell and Thomas Friedman for helpful comments on the manuscript. We are particularly grateful to the subjects who participated in this study.

1 Dworzynski K, Remington A, Rijsdijk F, Howell P, Plomin R: Genetic etiology in cases of recovered and persistent stuttering in an unselected, longitudinal sample of young twins. Am J Speech Lang Pathol 2007; 16: 169-178.

2 Fagnani C, Fibiger S, Skytthe A, Hjelmborg JV: Heritability and environmental effects for self-reported periods with stuttering: a twin study from Denmark. Logoped Phoniatr Vocol 2011; 36: 114-120.

3 Felsenfeld S: Finding susceptibility genes for developmental disorders of speech: the long and winding road. J Commun Disord 2002; 35: 329-345.

4 Ooki S: Genetic and environmental influences on stuttering and tics in Japanese twin children. Twin Res Hum Genet 2005; 8: 69-75.

5 Yairi E, Ambrose N, Cox N: Genetics of stuttering: a critical review. J Speech Hear Res 1996; 39: 771-784.
6 Kang C, Riazuddin S, Mundorff J et al: Mutations in the lysosomal enzyme-targeting pathway and persistent stuttering. N Engl J Med 2010; 362: 677-685.

7 Cathey SS, Leroy JG, Wood T et al: Phenotype and genotype in mucolipidoses II and III alpha/beta: a study of 61 probands. J Med Genet 2010; 47: 38-48.

8 Kudo M, Brem MS, Canfield WM: Mucolipidosis II (I-cell disease) and mucolipidosis IIIA (classical pseudo-hurler polydystrophy) are caused by mutations in the GIcNAcphosphotransferase alpha / beta -subunits precursor gene. Am J Hum Genet 2006; 78: 451-463.

9 Liu S, Zhang WM, Shi HP, Meng Y, Qiu ZQ: Three novel homozygous mutations in the GNPTG gene that cause mucolipidosis type III gamma. Gene 2014; 535: 294-298.

10 Paik KH, Song SM, Ki CS et al: Identification of mutations in the GNPTA (MGC4170) gene coding for GlcNAc-phosphotransferase alpha/beta subunits in Korean patients with mucolipidosis type II or type IIIA. Hum Mutat 2005; 26: 308-314.

11 Tiede S, Storch S, Lubke T et al: Mucolipidosis II is caused by mutations in GNPTA encoding the alpha/beta GlcNAc-1-phosphotransferase. Nat Med 2005; 11: 1109-1112.

12 Riley GD: Stuttering Severity Instrument for Children and Adults (SSI-3), 3rd edn. Los Angeles, CA, USA: Western Psychological Services, 1994, pp 1-33.

13 Webster RL: Evolution of a target-based behavioral-therapy for stuttering. J Fluency Disord 1980; 5: 303-320.

14 Pena SDJ, Di Pietro G, Fuchshuber-Moraes $\mathrm{M}$ et al: The genomic ancestry of individuals from different geographical regions of Brazil is more uniform than expected. PLoS One 2011; 6: e17063.

15 Bloodstein O, Ratner NB: A Handbook on Stuttering, 6th edn. Clifton Park, NY, USA: Cengage Learning, 2008, pp 78-91. 